\title{
Alloplasmic wheat lines, their photosynthetic activity and drought-tolerance
}

\author{
Terletskaya N.V. ${ }^{*}$, Salina E.A. ${ }^{2}$, Nesterov M.A. ${ }^{2}$, Zorbekova A.N. ${ }^{1}$, Altayeva N.A. ${ }^{1}$ \\ ${ }^{1}$ Institute of Plant Biology and Biotechnology, Almaty, Kazakhstan \\ ${ }^{2}$ Institute of Cytology and Genetics, SB RAS, Novosibirsk, Russia \\ *e-mail:teni02@mail.ru
}

They are investigated morphophysiological and photosynthetic indicators of response to drought-stress of nine wheat alloplasmic lines carrying the cytoplasm of tetraploid species $T$. dicoccum Shuebl. They were noted and significant differences in the regulation of growth and photosynthetic activity of alloplasmic lines between themselves under drought conditions and differences from euplasmic parental forms were shown. The most tolerant to drought alloplasmic lines D-d-05 b, D-b-05, D-41-05 and the most sensitive D-f-05 have been identified.

The use of 21 SSR (simple sequences repeats) markers for evaluation of genomic polymorphism of the wheat alloplasmic lines and the cv. Mironovskaya-808, used as the paternal form in crossing, showed that level of polymorphism for 7 lines and Mironovskaya- 808 does not exceed $15 \%$. The lowest level of polymorphism (less than $5 \%$ ) was observed between the lines D-b-05 and D-a- 05 .

The D-n-05 line is distinguished because it differs from the studied lines in 9 SSR fragments out of 21. Of interest is the D-f-05 line, since it, unlike other lines, identifies T. dicoccum genetic materal marked with Xgwm357 (1AL chromosome), Xgwm192c (4BS) and Xgwm 155 (3AL).

Obviously, selection on the basis of productivity and grain quality for 7 years by Dr. Khailenko, also led to the selection of wheat alloplasmic lines in which the presence of $T$. dicoccum nuclear genes is necessary to maintain high productivity based on nuclearcytoplasmic interactions, which compensates for its most sensitive to drought on the studied parameters, relative to other lines.

Thus, it was showed that the combination the nucleus and cytoplasm originating from parental forms belonging to different species can both improve and worsen the stressresistance and photosynthetic activity of wheat alloplasmic lines.

Acknowledgements: The work is supported by the granting program of the MES RK (AP05131734). 